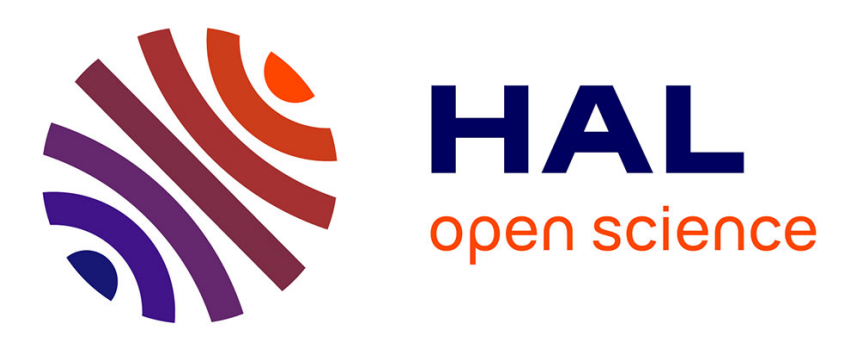

\title{
Contrôler, enfermer, éloigner. La répression policière et administrative des Algériens de Métropole (1946-1962)
}

Emmanuel Blanchard

\section{To cite this version:}

Emmanuel Blanchard. Contrôler, enfermer, éloigner. La répression policière et administrative des Algériens de Métropole (1946-1962). Raphaëlle Branche, Sylvie Thénault. La France en Guerre, 1954-1962, Autrement, pp.382-395, 2008, Collection "Mémoires Histoire". hal-00559762

\section{HAL Id: hal-00559762 \\ https://hal.science/hal-00559762}

Submitted on 31 Jan 2011

HAL is a multi-disciplinary open access archive for the deposit and dissemination of scientific research documents, whether they are published or not. The documents may come from teaching and research institutions in France or abroad, or from public or private research centers.
L'archive ouverte pluridisciplinaire HAL, est destinée au dépôt et à la diffusion de documents scientifiques de niveau recherche, publiés ou non, émanant des établissements d'enseignement et de recherche français ou étrangers, des laboratoires publics ou privés. 
35. CONTRÔLER, ENFERMER, ÉLOIGNER.

LA RÉPRESSION POLICIÈRE ET ADMINISTRATIVE DES ALGÉRIENS
DE MÉTROPOLE (1946-1962)
Emmanuel Blanchard

Entre 1944 et 1947, un ensemble de textes a organisé la reconnaissance de la citoyenneté des colonisés d'Algérie et l'égalité des droits entre les « musulmans » et les «Européens ${ }^{1}$ ». Le double collège électoral a singulièrement limité la portée de ces dispositions dans les départements d'Algérie, mais elles ont eu des conséquences très importantes pour les candidats à l'émigration. Citoyens à part entière à partir du moment où ils pénétraient dans l'Hexagone, ils bénéficiaient d'une entière liberté de circulation entre les deux rives de la Méditerranée. Celle-ci avait déjà été reconnue à divers moments de l'entre-deux-guerres, mais la conjoncture économique, marquée à la fois par la grande paupérisation des fellahs et l'abaissement des coûts de transport, lui a donné une portée inédite à partir de la fin de l'année 1946. Les arrivées en métropole sont alors devenues massives - avec un solde migratoire annuel moyen d'environ 20000 entre 1947 et 1962 -, alors même que les Algériens étaient au nombre des migrants que les experts souhaitaient ne pas voir s'installer en France ${ }^{2}$. Cette «immigration imposée ${ }^{3}$ » par le contexte

1. Laure Blevis, «Les avatars de la citoyenneté en Algérie coloniale ou les paradoxes d'une catégorisation ", Droit et société, nº 48, 2001, p. 557-580.

2. Patrick Weil, La France et ses étrangers. L'aventure d'une politique de l'immigration, 1938-1991, Paris, Calmann-Lévy, 1991, p. 54-59.

3. Georges Mauco formula cette distinction entre «immigration voulue» et " immigration imposée », à la veille de la Seconde Guerre mondiale, pour distinguer les «travailleurs» des « réfugiés». Patrick Weil, « Georges Mauco, expert en immigration : ethnoracisme et antisémitisme fielleux», in Pierre-André Taguieff 
colonial n'a pas tardé à susciter des protestations. De la fin de la Seconde Guerre mondiale jusqu'au dénouement de la guerre d'indépendance algérienne, la hiérarchie policière n'a eu de cesse de lutter contre ce «mythe de l'égalité des droits ${ }^{4}$ ". Avec un succès croissant, notamment en raison des répercussions métropolitaines de la guerre d'indépendance, elle a réclamé des mesures lui permettant de contrôler, d'enfermer et d'éloigner les Algériens sans entrave juridique ni contrôle du pouvoir judiciaire.

\section{Limiter la mobilité, une constante du contrôle policier des Algériens}

La liberté de circulation accordée dans le cadre de la défense de l'empire colonial est venue s'opposer aux principes de l'ordre public en métropole tels qu'ils étaient interprétés par les forces de l'ordre. La «clochardisation $^{5}$ " de nombreux migrants d'Algérie et la délinquance de subsistance de certains d'entre eux alimentent ainsi le flot des récriminations d'administrés et des campagnes de presse qualifiées de « racistes» par les rares défenseurs des Algériens ${ }^{6}$. Elles renforcent des stéréotypes coloniaux que la nouvelle donne juridique et politique ne suffit pas à affaiblir et permettent à la police d'essayer de récupérer les pouvoirs perdus à la Libération. Face au refus des autorités politiques de revenir sur la liberté de circulation, la hiérarchie policière a essayé de la limiter par divers artifices, dont de nombreux avaient déjà été mis en œuvre dans l'entre-deux-guerres. L'obligation de subir des examens médicaux et des vaccinations, de réunir un cautionnement équivalent aux éventuels frais de rapatriement et de posséder une pièce d'identité uniformisée avec photographie est ainsi évoquée comme un moyen

(dir.), L'Antisémitisme de plume, 1940-1944. Études et documents, Paris, Berg international éditeurs, 1999, p. 267-276.

4. Agendas de Roger Léonard, préfet de police (1947-1951), 24 novembre 1948. Fonds Léonard du Centre d'histoire de Sciences-Po, cote RL 1.

5. Germaine Tillion, Algérie 1957, Paris, Minuit, 1957, p. 40-41.

6. Ce sont en particulier les communistes qui, dans leur presse, dénonçaient les journaux « racistes ». L'Algérien en France était en pointe dans ces dénonciations. À propos de cette publication, voir l'article «Le rendez-vous manqué des Algériens et du Parti communiste français. L'expérience de L'Algérien en France (1950-1960)», p. xxx. 
d'éviter le départ des plus pauvres. Les nécessités de ne pas contrevenir à la Constitution, de tenir compte des représentants politiques des «Français musulmans d'Algérie », les concurrences et l'inertie administratives ont fait qu'avant le début de la guerre aucune disposition n'est venue véritablement limiter cette liberté de circulation.

Faute de contrôler l'émigration algérienne, la police a cherché à limiter ses possibilités de mobilité en métropole par deux biais : l'organisation de leur moindre visibilité dans l'espace public et celle des refoulements en Algérie. Pour ce faire, elle a eu abondamment recours au répertoire d'actions de la police des «indésirables $^{7}{ }$. Hors de tout cadre juridique se sont alors installées dans la durée des méthodes d'action qui n'avaient rien d'inédites. De rafles en internements dans des locaux de police, de fichages en refoulements, de contrôles d'identité en rétentions administratives, les Algériens partagent le sort des autres " citoyens diminués ${ }^{8}$ " (prostitués, vagabonds, homosexuels...) et d'un sous-prolétariat national dont le contrôle a longtemps constitué l'essentiel du travail policier ${ }^{9}$. Leur statut les protège néanmoins de mesures telles que l'expulsion et le refoulement, qui menacent les étrangers contrevenant à l'ordre public ou ne répondant pas aux attentes des employeurs. Les Algériens n'ont pas eu non plus à connaître les affres des internements administratifs de longue durée. Ces privations de liberté à la seule initiative de la police sont pourtant pratiquées dans l'illégalité la plus totale à l'égard d'autres nationaux, tels les vagabonds conduits à la Maison départementale de Nanterre ou les prostituées hospitalisées à l'ancienne prison Saint-Lazare. Les représentants et les soutiens politiques des colonisés d'Algérie sont alors suffisamment entendus pour que ce type de mesure ne leur soit pas appliqué. La hiérarchie policière ne cesse pourtant pas de se référer à un horizon internemental qui ne deviendra réalité que quelques années plus tard.

7. Emmanuel Blanchard, «L'encadrement des Algériens de Paris (1944-1954), entre contraintes juridiques et arbitraire policier », Crime, histoire \& sociétés, vol. XI, n ${ }^{\circ} 1,2007$, p. 5-25.

8. L'expression " citoyens diminués » est souvent utilisée par des représentants du PCF pour qualifier les Algériens de métropole. Voir notamment les interventions d'élus PCF aux conseils de Paris et de la Seine: Bulletin municipal officiel-conseil municipal, 18 juillet 1947, p. 342 ; Bulletin municipal officiel-conseil général, 20 mars 1953, p. 170.

9. Philippe Robert, Le Citoyen, le crime, l'État, Genève, Droz, 1999, p. 23-35.

384 - LA FRANCE EN GUERRE 1954-1962 
Malgré ces limites à l'arbitraire policier, les Algériens sont loin de bénéficier d'un traitement préférentiel. Ils sont l'objet de multiples conduites au poste, tant le week-end, afin d'éviter que la visibilité de ces travailleurs de l'ombre n'indispose les autres promeneurs parisiens ${ }^{10}$, qu'au moment des appels à mobilisation du Mouvement pour le triomphe des libertés démocratiques (MTLD). La répression des manifestations nationalistes indique même à quel point la violence policière à l'encontre des colonisés n'est pas cantonnée aux territoires ultramarins ${ }^{11}$. Il n'en reste pas moins qu'à cette époque le «chèque en gris ${ }^{12}$ " accordé par le pouvoir politique à une police chargée du maintien de l'ordre social et colonial, même au prix d'entorses à la légalité, n'est pas un blanc-seing : un certain nombre de contrepoids limitent l'arbitraire policier. Ils s'affaiblissent néanmoins considérablement lorsque se font sentir les répercussions métropolitaines de la guerre d'Algérie.

\section{Des désirs policiers exaucés grâce à la guerre}

À partir de 1955, la préfecture de police de Paris voit ainsi acceptées la plupart des revendications pour lesquelles elle s'était heurtée, les années précédentes, au refus du ministère de l'Intérieur et plus généralement $\mathrm{du}$ pouvoir politique.

Les Algériens sont ainsi progressivement fichés et mis en cartes. Le fichier des "Nord-Africains", détruit en 1940 afin de ne pas tomber dans les mains de l'occupant allemand, n'avait pas été remplacé à la Libération. Pour pallier cette lacune, la préfecture de police de Paris a mené, sous couvert d'enquêtes sociales et de recensements, de

10. Léo Bogart, "Les Algériens en France, adaptation réussie et non réussie », in Ined, Français et immigrés, Paris, PUF-Ined, 1954, p. 48.

11. De 1937 à 1962, les seuls manifestants tués à Paris par les forces de l'ordre furent des Algériens (28 mai 1952, 14 juillet 1953). À ces sept victimes des forces de l'ordre doivent être ajoutées celles (au moins trois) qui furent tuées en province, particulièrement en mai 1952. Danielle Tartakowsky, Les Manifestations de rue en France, Paris, Fayard, p. 633-635.

12. Nous empruntons ce concept au criminologue canadien Jean-Paul Brodeur qui l'utilise à propos des situations dans lesquelles les pouvoirs publics laissent la police libre de ses modalités de répression, même extralégales, tant que n'éclatent pas de controverses politiques. Jean-Paul Brodeur, «La police, mythes et réalités », Criminologie, vol. XVII, nº 1, 1984, p. 9-41. 
nombreuses opérations visant à une meilleure connaissance des « milieux nord-africains ». À partir d'août 1953, la Brigade des agressions et violences (BAV) ${ }^{13}$ multiplie les rafles, parfois avec l'appui de photographes, afin d'alimenter un nouveau fichier. Il reste très incomplet, la préfecture de police ne disposant d'aucune prérogative légale obligeant tous les Algériens à prendre contact avec elle. Avec l'entrée dans la guerre, cette dimension limitée du fichage devient un désavantage que ne peuvent plus contrebalancer les bénéfices politiques attendus d'un relatif respect du statut des Algériens. C'est à cette lumière que doivent principalement être analysées des mesures telles que la création de la carte nationale d'identité en octobre 1955. En mars 1956, quand le nouveau régime de circulation entre les deux rives de la Méditerranée est institué ${ }^{14}$, la carte nationale d'identité devient obligatoire pour obtenir une autorisation de voyage. Ces textes législatifs ne peuvent être complètement appliqués en raison notamment de contraintes administratives et bureaucratiques, comme la tenue de l'état civil en Algérie, les effectifs policiers disponibles... Ils visent principalement à obliger les Algériens à se faire connaître des services de police. À partir de 1958, la préfecture de police de Paris et la Sûreté nationale reconnaissent explicitement que l'enregistrement de l'ensemble des Algériens est un objectif prioritaire. Pour la première, cette ambition s'accompagne de la mise en place d'un appareil militaro-policier, les Services d'assistance technique aux Français musulmans d'Algérie (SAT-FMA) ${ }^{15}$, qui entendent subordonner toute action sociale aux impératifs du maintien de l'ordre et de la "guerre contre-révolutionnaire».

Les forces de l'ordre obtiennent aussi satisfaction en matière de contrôle des mouvements, d'assignation à résidence et de refoulement des «indésirables». En septembre 1958 et octobre 1961, la promulgation de couvre-feux limités aux seuls «travailleurs algériens » est sans doute la mesure la plus emblématique de ce retournement du rapport

13. Emmanuel Blanchard, «Police judiciaire et pratiques d'exception pendant la guerre d'Algérie ", Vingtième Siècle. Revue d'histoire, nº 90, avril-juin 2006, p. 61-72.

14. À compter de cette date, la libre circulation est supprimée et il est nécessaire d'obtenir une autorisation de voyage, délivrée dans les commissariats de police, pour se rendre en Algérie.

15. Sur les SAT-FMA dont les personnels et méthodes sont importés d'Algérie: Jim House, Neil MacMaster, Paris 1961 : Algerians, State Terror and Post-colonial Memories, Oxford, Oxford University Press, 2006, p. 66-77.

386 - LA FRANCE EN GUERRE 1954-1962 
de forces entre la police et les organes politiques et juridiques censés la contrôler ${ }^{16}$. Symboliquement au moins ${ }^{17}$, la police parisienne obtient, au mépris du droit, certaines des prérogatives en matière de limitation de la liberté de circuler qu'elle réclamait depuis de nombreuses années.

Dans la même optique de limitation de la liberté de mouvement de «suspects", les autorités préfectorales ont aussi recours à l'assignation à résidence à domicile: les individus visés doivent se présenter toutes les semaines au commissariat de leur localité pour faire viser leur carnet d'assignation à résidence. Les autorités policières savent que cette mesure n'est pas d'une grande efficacité et lui préfèrent les nouvelles possibilités d'enfermement. En 1957-1958, deux textes autorisent ainsi en métropole l'internement administratif, pratiqué en Algérie dès avril 1955 après le vote de l'état d'urgence. La loi du 26 juillet 1957 étend à la métropole une partie des pouvoirs spéciaux adoptés pour l'Algérie le 16 mars 1956. Elle ouvre ainsi la possibilité d'assigner à résidence toute personne condamnée en application des «lois sur les groupes de combat et milices privées ». Cette assignation, qui se concrétise par un internement dans l'un des quatre «centres d'assignation à résidence surveillés » (CARS) ${ }^{18}$ ouverts dans la foulée, est donc conditionnée à une condamnation judicaire préalable. Elle permet au ministère de l'Intérieur de détenir de façon illimitée les «individus dangereux » libérés de prison.

Malgré ces nouveaux pouvoirs, la police ne se satisfait pas de rester sous la dépendance de l'autorité judiciaire. Le «laxisme » de cette dernière, régulièrement dénoncé en temps de paix, apparait insupportable à la hiérarchie policière alors que l'unité et l'intégrité nationales sont remises en cause par une "rébellion ». La nomination de Maurice Papon à la tête de la préfecture de police de Paris est un des facteurs qui a permis que des moyens nouveaux lui soient accordés. Un des projets de

16. Sylvie Thénault, «Les couvre-feux en région parisienne pendant la guerre d'Algérie », à paraître.

17. Ces textes furent surtout importants pour limiter la circulation nocturne des véhicules conduits par des Algériens, dont ils permettaient la saisie sans autre forme de procès. Pour celle des piétons, les couvre-feux ne faisaient que renforcer les pratiques policières de contrôle nocturne de tous les Algériens circulant seuls ou en groupe.

18. Sylvie Thénault, "Personnel et internés dans les camps d'internement de la guerre d'Algérie. Entre stéréotypes coloniaux et combat pour l'indépendance ", Politix, nº 69, 2005, p. 63-81. 
Papon inspire grandement l'ordonnance du 7 octobre 1958 qui donne de nouveaux pouvoirs aux autorités policières et administratives en matière d'internement. Ce nouveau texte élargit considérablement le champ des individus susceptibles de faire l'objet d'une mesure d'assignation à résidence : toutes les «personnes dangereuses pour la sécurité publique en raison de l'aide matérielle directe ou indirecte qu'elles apportent aux rebelles des départements algériens » sont concernées.

Des précisions ultérieures confirment cependant que seuls les «Français musulmans d'Algérie » sont visés par ces mesures privatives de liberté dont la promulgation est grandement facilitée. L'ordonnance prévoit en effet qu'un simple arrêté préfectoral, signé sur la foi de rapports des RG, suffit à placer les individus «à surveiller » dans des établissements pénitentiaires ou des "centres de triage ${ }^{19}$ » qui sont créés dans les départements où la "colonie nord-africaine » est fortement implantée. Seule véritable limite à ces pouvoirs exorbitants accordés aux autorités policières : cet internement préfectoral ne peut normalement pas dépasser quinze jours. Il peut cependant être prolongé par un arrêté ministériel d'assignation à résidence, sans limitation de durée. Ce dernier est pris en fonction des seuls rapports de police. La police ne voit d'ailleurs guère ses pouvoirs limités par la création de la Commission de vérification des mesures de sécurité publique prévue à l'article 3 de l'ordonnance du 7 octobre 1958. Même si certains de ses avis transmis au ministère de l'Intérieur sont suivis d'effets, elle n'exerce qu'un vague contrôle a posteriori et ne joue qu'un rôle consultatif. Son rôle est donc tout aussi limité que celui de la Commission de sauvegarde des droits et libertés individuels chargée d'enquêter sur les tortures et les disparitions en Algérie $^{20}$.

19. Les centres de triage étaient des lieux d'enfermement et parfois d'interrogatoire des individus assignés à résidence par les préfets pour une durée de quinze jours. Parfois dits aussi «centres de transit» ou «centres d'identification», le plus souvent caractérisés par une simple adresse pour cacher leur véritable usage, ils étaient généralement situés dans l'enceinte des commissariats ou autres locaux de police.

20. Au sujet de ces commissions : Raphaëlle Branche, "La Commission de sauvegarde pendant la guerre d'Algérie : chronique d'un échec annoncé », Vingtième Siècle. Revue d'histoire, $\mathrm{n}^{\circ} 61$, janvier-mars 1999, p. 14-29. De la même auteure : "La seconde Commission de sauvegarde des droits et libertés individuels ", in AFHJ, La Justice en Algérie, 1830-1962, La Documentation française, 2005, p. 237-246.

388 • LA FRANCE EN GUERRE 1954-1962 
À l'issue du délai imposé par l'arrêté préfectoral d'assignation à résidence, les individus qui font l'objet d'un arrêté ministériel sont transférés dans un des quatre CARS. Devant la volonté policière de placer en rétention le maximum d'individus, les difficultés de coordination entre de multiples ministères (Justice, Intérieur, Défense ${ }^{21}$ ), le coût de cette politique et l'absence de respect des délais de signature par le ministère de l'Intérieur sont les seules limites au recours aux assignations à résidence «sans durée préfixe». Ces considérations incitent ainsi Émile Pelletier, ministre de l’Intérieur, à rappeler en octobre 1958 :

Il ne saurait être question pour le moment, bien qu'elle puisse apparaître comme un moyen commode de se débarrasser de tous les éléments FSNA (Français de souche nord-africaine(tenus pour dangereux, de recourir à une politique massive d'internement, en raison des capacités très réduites qu'offrent encore, tant en métropole qu'en Algérie, les centres d'hébergement destinés à les recevoir ${ }^{22}$.

Ce dispositif est cependant monté en charge dans les années suivantes. Pendant près de trois ans, il a pris le pas sur les velléités de renvoyer les « suspects » outre-Méditerranée.

\section{L'Algérie, terre de bannissement}

Les « rapatriements » dans les départements algériens sont l'autre horizon du travail policier. Dès la fin des années 1940, les dispositions relatives à la reconduite des vagabonds dans leur département ou pays d'origine ont été invoquées et utilisées comme un moyen de se débarrasser de certains « oisifs ». Les dispositions réglementaires en la matière sont modifiées à plusieurs reprises afin d'élargir les catégories d'individus concernés par des dispositifs gérés par différents ministères. Pour les Algériens, citoyens français, ces retours sont censés n'être possibles que si ceux-ci en ont explicitement exprimé la volonté. La préfecture

21. La gendarmerie était chargée des transferts des assignés à résidence des centres de triage aux CARS.

22. Circulaire $\mathrm{n}^{\circ} 577 \mathrm{du}$ ministère de l'Intérieur relative à l'application de l'ordonnance du 7 octobre 1958 et envoyée à tous les préfets le 15 octobre 1958. 
de police de Paris est pourtant tentée de leur appliquer les dispositions relatives aux étrangers qui peuvent être reconduits contre leur gré. Si des retours forcés sont effectivement mis en œuvre, ils connaissent cependant une application limitée, notamment pour des questions de répartition de la charge financière de ces rapatriements - les communes et départements algériens devant contribuer au frais et n’y étant généralement pas enclins. Ces dispositions connaissent cependant un certain succès, puisque le nombre des rapatriements est multiplié par dix entre 1950 et 1954, pour atteindre un total de 6500 au cours de cette seule année ${ }^{23}$. Ces retours n'augmentent pas beaucoup durant la guerre d'Algérie. Leur nombre est limité par l'implication de différents ministères et le coût de la prise en charge des transports. Cependant, lorsqu'il apparaît nécessaire de signifier que le pouvoir sait être ferme avec les nationalistes, des « reconduites au douar d'origine » par centaines sont opérées sans qu'aucune disposition juridique quelconque soit invoquée. Il en a été ainsi après les émeutes de la Goutte-d'Or en août $1955^{24}$ et après les manifestations d'octobre 1961.

L'ordonnance du 7 octobre 1958 ouvre aussi la possibilité de recourir à l'«éloignement, sorte d'interdiction de séjour administrative $^{25}$ ». Si, traditionnellement, cette dernière mesure est peu efficace, sinon à transformer les «interdits de séjour » qui ne la respectent pas en indicateurs de police, $l^{\prime}$ « éloignement» ouvre la voie à de nouveaux départs contraints vers l'Algérie. Les interdictions portent le plus souvent sur la seule région parisienne, mais peuvent être étendues à l'ensemble du territoire français.

Dans ce cas, il semble qu'en règle générale ces interdits de séjour ne soient pas reconduits jusqu'en Algérie. En revanche, les demandes d'autorisations de voyage sont parfois utilisées pour favoriser l'« élimination » d'individus fichés $Z$ dans la catégorie «très dangereux ». Ce procédé répond en outre à la nécessité de limiter les coûts de la répression, puisque les intéressés financent eux-mêmes leur voyage. Quand ils demandent à rentrer en Algérie et déposent leur dossier auprès du commissariat de leur localité, l'autorisation de voyager leur est accordée et dotée d'un numéro avec la mention du département de résidence en

23. Archives nationales (AN) F1A 5134.

24. André-Louis Dubois et Pierre Sergent, Le Malentendu algérien. 12 ans après, Paris, Fayard, 1972, p. 106-107.

25. Circulaire du 15 octobre 1958, op. cit.

390 - LA FRANCE EN GUERRE 1954-1962 
métropole. Ce signalement doit entraîner l'arrestation de son possesseur à l'arrivée en Algérie. Si tout fonctionne bien, la personne en question a ainsi payé son voyage à destination d'un camp algérien où elle se retrouve assignée à résidence. Cette procédure a été en vigueur d'août 1957 à avril 1959. Quelques « incidents regrettables » ont mis fin à cette expérience : il est arrivé, par exemple, que des individus signalés fassent l'aller-retour sans être inquiétés ou que des personnes soient arrêtées à leur débarquement en Algérie sans raison. Par la suite, «sauf motifs humanitaires sérieux », traités au niveau ministériel, les demandes de sortie du territoire français ne sont plus accordées aux fichés $Z^{26}$.

En Algérie, les autorités locales, bien que dotées de pouvoirs de police étendus et renforcées par les effectifs de l'armée, n'ont pas intérêt à ce que les militants frontistes les plus aguerris soient concentrés sur leur territoire, même enfermés dans des camps qui s'avèrent être de véritables écoles des cadres du FLN. Alors que, pendant des années, elles ont abondamment été accusées d'encourager l'émigration en métropole des «mauvais éléments » dont elles voulaient se débarrasser, elles ont dû, à leur tour, faire face à des arrivées qu'elles n'avaient pas souhaitées. Ces luttes d'influence entre administrations métropolitaines et algériennes font partie des raisons qui expliquent que, tout au long de la guerre d'Algérie, les circuits de la répression administrative et juridique ont connu plusieurs inflexions.

\section{Permanence et évolution des circuits répressifs ${ }^{27}$}

Au début de la guerre d'indépendance, ces circuits aboutissent presque systématiquement outre-Méditerranée. Pour les forces de l'ordre, l'Algérie est en effet le débouché naturel pour sanctionner la venue

26. Télégrammes secrets du ministère de l’Intérieur, 7 janvier et 28 avril 1959 , archives de la préfecture de police de Paris (APP) HA 8. Sur le fichier Z, voir l'article «Identifier les nationalistes algériens : les fiches $\mathrm{Z}$ », $\mathbf{p} . \mathbf{x x}$.

27. Cette partie est surtout fondée sur le dépouillement des archives de la répression des nationalistes algériens en Seine-et-Oise conservées aux archives départementales (ADSO) 78 - les archives de Seine-et-Oise se trouvent désormais aux archives départementales des Yvelines. Les dossiers de police de 184 militants de la région d'Argenteuil pour lesquels des demandes d'assignation à résidence avaient été faites au ministère de l'Intérieur entre 1957 et 1961 ont été analysés. ADSO 781 W 511 à 1 W 547.

35. CONTRÔLER, ENFERMER, ÉLOIGNER. LA RÉPRESSION POLICIÈRE ET ADMINISTRATIVE • 391 
$\mathrm{d}^{\prime}$ « indésirables » qui n'auraient pas dû quitter leurs départements d'origine. Surtout, les moyens juridiques de répression y sont plus étendus que les seules ressources du Code pénal et du Code d'instruction criminelle auxquels les forces de l'ordre de métropole sont censées se conformer. La coopération avec des juges d'Algérie permet de contourner ces limites. À partir de 1957, des opérations sont lancées au plan national afin de déférer devant la justice algérienne des militants FLN de métropole sur la base du délit d'atteinte à la sûreté extérieure de l'État et de leurs liens (financiers notamment) avec une organisation dont les agissements outre-Méditerranée font l'objet de poursuites judiciaires. Arrêtés sur la base de commissions rogatoires lancées par des juges algérois, des militants parisiens sont ainsi transférés en Algérie. Certains de ces inculpés y sont emprisonnés, d'autres font l'objet d'un non-lieu suivi d'une assignation à résidence dans l'un des camps déjà ouverts, mais la plupart ne tardent pas à rentrer en métropole. La fragilité des attendus juridiques qui ont présidé à leurs poursuites devant une juridiction algérienne, les libérations successives de camps surpeuplés, les réticences des autorités des départements algériens à accueillir ces suspects ont joué en faveur de ces retours. Cet échec relatif d'une exportation de la répression dans le cadre colonial a incité les autorités policières à plaider pour un renforcement de leurs prérogatives et des possibilités de répression administrative et judiciaire en métropole.

Les allongements successifs des durées de garde à vue et les possibilités de perquisition - notamment nocturne - sans contrôle d'un juge $\mathrm{d}^{\prime}$ instruction sont venus considérablement renforcer l'arsenal policier ${ }^{28}$. Grâce aux alinéas de l'ordonnance du 7 octobre 1958 relatifs à l'internement administratif, les forces de l'ordre sont de plus en plus affranchies de tout contrôle et débouché judiciaires. L'enfermement dans des camps fait en effet office de sanction idéale pour les autorités policières. Cette absence d'instructions et de poursuites judicaires permet que les affaires menées en dépit du respect de toute procédure se traduisent quand même par l'enfermement des principaux mis en cause. Seules les poursuites pour lesquelles les règles formelles ont été un tant soit peu prises en compte et des preuves d'appartenance au FLN ou participation à des délits de droit commun (violences, racket...) réunies sont portées devant les juridictions, en correctionnelle le plus souvent. [sens ?

28. Stéphane Boittiaux, La Procédure de répression des infractions terroristes depuis le 30 octobre 1954, thèse de droit, université de Paris, 1962.

392 - LA FRANCE EN GUERRE 1954-1962 
«Seules les poursuites pour lesquelles les règles formelles ont été un tant soit peu prises en compte et l'appartenance au FLN ou la participation à des délits de droit commun (violences, racket...) sont portées devant les juridictions, en correctionnelle le plus souvent » ?]. De surcroît, le 8 octobre 1958, une ordonnance vient étendre la possibilité de saisir la justice militaire en métropole. Celle-ci n'intervient pratiquement qu'au stade du jugement et, à partir de 1960, l'instruction est totalement laissée entre les seules mains du pouvoir policier $^{29}$. Mais si les autorités policières attendent des tribunaux permanents des forces armées (TPFA) une plus grande sévérité que des juges civils, leurs attentes sont, semble-t-il, en partie déçues. Bien que seules les affaires les plus graves soient déférées devant les TPFA, ils ne s'estiment pas toujours compétents et prononcent des peines qui, en moyenne, ne diffèrent guère de celles des tribunaux civils ${ }^{30}$. Quelquefois, pourtant, ils n'hésitent pas à rendre plusieurs verdicts de condamnation à mort en un seul après-midi ${ }^{31}$. Ces verdicts expéditifs incitent la préfecture de police de Paris à demander que des affaires toujours plus nombreuses puissent être directement déférées devant les juridictions militaires $^{32}$.

Le laxisme supposé de la justice, qui est à l'origine de ces innovations juridiques et de l'extension inédite des pouvoirs de police, est pourtant loin d'être avéré. Tout au long de la guerre d'Algérie, en moyenne plus de 10000 Algériens ont été détenus dans des prisons métropolitaines qui n'auraient pu supporter une nouvelle hausse de la population carcérale ${ }^{33}$. Peut-être est-ce de ce fait, ou de la seule volonté de ne pas s'écarter de leur strict mandat professionnel et de ne pas gêner

29. Ordonnance 60-520 du 3 juin 1960. Sur le sujet, voir Jean-Marc Berlière, "Policiers et pouvoir politique en période de crise : la guerre d'Algérie (1958-1962) ", in Être policier : les métiers de police(s), XVIII'-XXe siècle, Rennes, PUR, à paraître.

30. Rapport du cabinet du ministère de l'Intérieur aux directeurs de la préfecture de police de Paris et de la Sûreté nationale, 18 mai 1960, APP HA 65,

31. Entretien avec Henri Leclerc (avocat, collaborateur d'Yves Dechezelle et de Pierre Stibbe, entre 1956 et 1959), Paris, le 19 mai 2005. Il évoque ici le TPFA du Cherche-Midi à Paris.

32. Pour des exemples de répartition et de luttes de compétences entre les juridictions civiles et militaires : Annie Deperchin et Arnaud Lecompte, « Les crimes commis par les Algériens en métropole devant la cour d'assises du Nord, 1954-1962 », in AFHJ, op. cit, p. 257-270.

33. Chiffres disponibles dans les comptes rendus des réunions des CTAM

35. CONTRÔLER, ENFERMER, ÉLOIGNER. LA RÉPRESSION POLICIÈRE ET ADMINISTRATIVE • 393 
le gouvernement, que les juges ne se sont jamais intéressés aux conditions d'internement administratif. En moyenne, ces assignations à résidence dans des camps ont concerné environ 5000 personnes pour les seuls CARS $^{34}$, soit, encore une fois, la totalité des places disponibles. Quant au nombre de personnes retenues dans les centres de triage, il est inconnu, et il est à noter que la durée d'internement dépendait plus des locaux disponibles et des possibilités d'assignation à résidence dans les CARS que de la limite de quinze jours imposée par les textes réglementaires ${ }^{35}$.

Les difficultés logistiques et plus encore les accusations portées contre les militants éloignés des grands centres urbains, soupçonnés de propager l'influence du FLN dans les villes ou campagnes où ils étaient assignés à résidence, ont fait revenir les forces de police aux stratégies de refoulement. Le caractère inéluctable de l'indépendance algérienne a eu pour effet que les problèmes politiques posés par le renvoi outreMéditerranée des «terroristes » arrêtés en métropole ne se posaient plus. À partir de 1961, seule compte l'« élimination des indésirables » dont la présence dans l'Hexagone n'est pas souhaitée. Les suites des arrestations opérées après le 17 octobre 1961 sont symptomatiques de cette évolution : au-delà de l'internement de près de 15000 manifestants dans des camps de fortune, comme par exemple le Centre d'identification de Vincennes, le Palais des Sports, le stade Pierre-de-Coubertin, le Parc des Expositions, dans lesquels de nombreuses violences ont été commises, aucune réponse judiciaire, par l'emprisonnement, ou administrative,

(1958-1962), AN F1A 5014. Ces comptages mêlent les détenus de droit commun et les détenus politiques. À la fin 1962, un peu plus de 3000 Algériens restaient emprisonnés en métropole.

34. Dans les comptes rendus des CTAM, cette rubrique ne fait son apparition qu'à la fin 1959, moment où le nombre d'internés atteint son maximum (5 700) pour se stabiliser ensuite à un peu moins de 5000 , puis décliner rapidement au quatrième trimestre 1961.

35. Les dossiers de police des internés de la caserne Noailles à Versailles (centre de triage pour le département de Seine-et-Oise) montrent que certains d'entre eux y restaient au-delà de la durée légale, mais rarement plus de quelques jours du fait de la surpopulation de ces locaux. À partir de 1959, avec l'ouverture du Centre d'identification de Vincennes (CIV), la préfecture de police disposa en la matière de plus de moyens et certains internés y restèrent enfermés plusieurs mois. Entretien avec Saad Abssi (Gennevilliers, le 3 octobre 2007), resté deux mois au CIV au début de l'année 1960.

394 - LA FRANCE EN GUERRE 1954-1962 
par l'assignation à résidence, n'a été apportée. Hors de tout cadre légal, plus de 1500 Algériens ont été « reconduits dans leur douar d'origine » à partir du 19 octobre et dans les semaines qui ont suivi ${ }^{36}$. Le caractère exceptionnel de ces manifestations et de leur répression a permis de mettre en œuvre ce que les forces de police exigeaient depuis des années : expulser sans autre forme de procès des « Français musulmans d'Algérie » que leur citoyenneté n'avait jamais préservés des mesures de police pratiquées dans le monde colonial ou de celles utilisées à l'encontre des étrangers de métropole.

36. Linda Amiri, La Bataille de France. La guerre d'Algérie en métropole, Paris, Robert Laffont, 2004, p. 167-170.

35. CONTRÔLER, ENFERMER, ÉLOIGNER. LA RÉPRESSION POLICIÈRE ET ADMINISTRATIVE • 395 